\title{
Hikikomori. Las voces silenciosas de la sociedad japonesa
}

\section{Hikikomori. The Silent Voices of Japanese Society}

DOI: 10.32870/mycp.v8i23.561
María Elena Romero ${ }^{1}$

\begin{abstract}
Resumen
El término hikikomori identifica tanto a un fenómeno como al individuo que tiende a aislarse socialmente y que afecta primordialmente a adolescentes o jóvenes que se encierran en sus habitaciones por seis o más meses. Este síndrome está siendo más estudiado desde la psicología; sin embargo las investigaciones incluyen los efectos que sobre este sector de la población tienen los cambios sociales que permean la sociedad japonesa de hoy. Elementos culturales, como la pertenencia al grupo, el honor y el éxito, contribuyen a crear un ambiente más complejo para la realización de los jóvenes, generando un sentimiento de frustración. En aras de no ser criticado o cuestionado sobre su desempeño, se aíslan. Es relevante hacer aquí una revisión de los estudios más conocidos sobre el tema, así como los factores culturales de Japón y las acciones emprendidas por el gobierno; considerando que este fenómeno no es exclusivo de la sociedad japonesa y que dadas las presiones sociales y la falta de oportunidades en la sociedad global actual, representa hoy un riesgo para la juventud en general.
\end{abstract}

Palabras clave: hikikomori, sociedad japonesa, desempleo, jóvenes, crisis.

\begin{abstract}
Hikikomori identifies both a phenomenon and a person who tends to socially isolate. It primarily affects adolescents or young people who lock themselves in their rooms for six or more months. This syndrome is being studied more from the psychology approach; nevertheless research includes the impact that social changes have had on Japanese youth today. Cultural elements, such as belonging to the group, honor, and success, contribute to create a more complex environment for the realization of young people, causing a feeling of frustration. Young people, in order not to be criticized or questioned about their performance, they rather isolate. It is important to review the most well-known studies on the subject, as well as the cultural factors of Japan and the actions undertaken by the Government; considering that this phenomenon is not exclusive to Japanese society and that given the social pressures and lack of opportunities in today's global society, it represents a risk for youth in general.
\end{abstract}

Keywords: hikikomori, Japanese society, unemployment, youth, crisis.

\section{Introducción}

Los jóvenes asumen día a día nuevos retos en un ambiente cada vez más competitivo. Ser mejores en la escuela o en el trabajo requiere de mayores

Artículo recibido el 26 de septiembre de 2018 y dictaminado el 18 de enero de 2019.

1. Universidad de Colima, Facultad de Ciencias Políticas y Sociales. . Carretera ColimaGuadalajara Km. 3.2m Col. El Diezmo, C.P. 28010, Colima, Colima, México. Orcid: http:// orcid.org/0000-0003-3550-9483 Correo electrónico: meromero@ucol.mx 
competencias y habilidades. Sin embargo, estar mejor preparados no les asegura mejores posiciones. La disminución de oportunidades hace que el reto, en ocasiones, provoque un sentimiento de frustración que los orilla al aislamiento social. No todos los jóvenes están preparados para asumir que el resultado de esforzarse no necesariamente llevará al éxito, algunos que no logran asumir esa realidad prefieren el aislamiento social a la crítica. Aislarse socialmente se ha convertido en un fenómeno cada vez más recurrente en Japón.

Casos como el de un adolescente de 14 años, quien repentinamente dejó la escuela sin motivo evidente y decidió no volver a salir durante dos años, son cada vez más constantes. En el caso de este joven, documentado por Teo y Gaw (2010), la intervención de la familia y una efectiva terapia psicológica lograron que se reincorporara a la sociedad.

Los análisis realizados en torno a esta reclusión han sido realizados desde la perspectiva psicológica, atendiendo factores mentales asociados con la bipolaridad, la depresión e incluso con la esquizofrenia. Sin embargo, como argumentan Teo y Gaw (2010), si bien es cierto que esta conducta responde a un desorden psicológico, también se han encontrado factores asociados a cambios sociales.

Desde la década de 1950 se han observado procesos de rechazo social, escolar y aislamiento. Pero fue en 1980 que se revisaron casos de rechazo escolar atribuibles a la dificultad de asumir los códigos impuestos por las instituciones educativas japonesas. En la década de 1990 se incrementó el número de estudios psicológicos que además sumaban factores sociológicos atribuibles a la crisis económica y cambios en la estructura social japonesa. Las aspiraciones de jóvenes egresados de universidades en las décadas de 1960 y 1970 se cumplieron en la medida que se incorporaron a los centros productivos y obtuvieron beneficios, como el trabajo vitalicio, que se cancelaban ante el estallido de la economía burbuja y la puesta en marcha de las reformas para reposicionar a Japón en la economía internacional.

La reclusión o aislamiento social es conocido en Japón como hikikomori, término que como dicen Suwa y Suzuki, (2013) se refiere tanto al individuo como al fenómeno en sí. Su importancia social y efectos en la población joven japonesa va en aumento, por ello es que aquí se propone la revisión de algunos de los textos más reconocidos sobre el tema, con el fin de visibilizar el fenómeno y entenderlo como un problema que se extiende en Japón, pero que también se ha localizado en otros países como Corea del Sur y en algunas naciones europeas. 
Los textos revisados contienen elementos psicológicos en su análisis; sin embargo, reconocen que los factores contextuales del Japón de hoy han propiciado un incremento en los casos de aislamiento social. Aquí se entiende el término hikikomori como un fenómeno de aislamiento o reclusión social, pero también se utiliza para denominar al individuo que sufre este síndrome. El objetivo de este trabajo es introducir la discusión sobre este fenómeno, mismo que se está extendiendo más allá de Japón y evidenciar la complejidad para definir sus causas.

El trabajo se divide en tres apartados. En el primero se recuperan los estudios más conocidos sobre el tema, rescatando sus hallazgos y aportaciones más importantes. En el segundo se presentan temas de índole cultural que se considera han afectado a la juventud japonesa y que la orillan al aislamiento; posteriormente se revisan algunas de las acciones emprendidas por el gobierno japonés, rescatando las encuestas realizadas a fin de reconocer la importancia del fenómeno y diseñar acciones concretas para atenderlo. Finalmente se presentan algunos comentarios finales a manera de conclusión.

\section{Estudios sobre el fenómeno hikikomori en Japón}

La reclusión o aislamiento social tiene expresiones asociadas a padecimientos tales como la esquizofrenia, la ansiedad, la depresión, entre otros; asimismo, atiende a contextos sociales de crisis que afectan el desempeño de los jóvenes, de manera que definir de forma determinante este padecimiento es complejo.

Los estudios que aquí se revisan analizan el tema desde el área de la psicología; sin embargo, todos discuten o mencionan factores sociales a los que atribuyen la decisión de los jóvenes de aislarse socialmente. Erikson (1950) en su obra Childhood and Society [Niñez y sociedad] analizó a jóvenes que se aíslan debido a la incapacidad para adaptarse a un ambiente de crisis. El aislamiento, dice, se enfatiza entre los 20 y 39 años cuando no han logrado superar la crisis y son incapaces se integrarse a la sociedad. De manera que no hablamos de un fenómeno nuevo, pero sí de un fenómeno al que se le ha prestado mayor atención y difusión y que se ha agudizado debido a las condiciones actuales en Japón.

Saito publicó en 1998 shakaitekihikikomori-owariwaranaishiharuki [Hikikomori. Adolescencia sin fin] que se tradujo al inglés en 2013 y a partir de entonces el término se popularizó. En su obra, Saito definió a un hikikomori como aquella persona que se recluye en su propio hogar por al menos un periodo de seis 
meses o más. Afirmó que es extremadamente difícil determinar a través de encuestas el número real de afectados, existiendo una enorme incapacidad para evidenciar el problema de una manera apropiada (Saito, 2013, p. 10). En este sentido, Saito afirma que se requiere profundizar el análisis y definir las condiciones especiales de un hikikomori, ya que en ocasiones el aislamiento social se atribuye a afecciones psicológicas que el autor identifica como "fobia social" o "desorden de personalidad evasiva"; en la medida que se avance en el análisis de un hikikomori, entonces habrá mejores posibilidades de tratamiento (2013, p. 76).

Furlong (2008) contribuye al estudio del fenómeno analizando tres factores sociales: 1) la familia; 2) la educación, y 3) el mercado laboral.

1. En el caso de la familia, afirma que un enfoque popular sobre hikikomori sugiere que las familias japonesas conservadoras se han convertido de alguna manera en disfuncionales y enfrentan problemas tratando de preparar a sus hijos para el contexto socioeconómico del Japón moderno. "Con la falta de apoyo estatal para los jóvenes, las familias esperan asumir la responsabilidad de su descendencia hasta que sean plenamente capaces de sostenerse sobre sus propios pies (Furlong, 2008, p. 314). La responsabilidad es recíproca, esperando que en la vejez, los padres sean atendidos por los hijos. En este sentido, argumenta Furlong (2008), la independencia no es tan valorada como en Occidente; los principios confucianos condicionan las relaciones familiares y exigen el respeto a los padres y el cuidado de por vida. Cuando los hijos, a pesar de las condiciones con las que fueron educados, no encuentran los medios para retribuir el cuidado a sus padres y siguen dependiendo de ellos, surge un sentimiento de frustración.

2. En el caso de la educación, el sistema educativo japonés es rígido y altamente exigente. Esta situación es muy apreciada porque implica una educación de excelencia que les permitirá a los jóvenes ubicarse con éxito en el campo laboral. Sin embargo, al egresar de programas de alto rendimiento que implicaron un gran esfuerzo, los jóvenes enfrentan la falta de oportunidades para obtener un empleo satisfactorio de acuerdo con su nivel de formación.

3. En relación con el mercado laboral, Furlong afirma que a pesar del reconocimiento que se hace al vínculo entre la transformación del mercado laboral y el fenómeno hikikomori, hay una tendencia a sobre-simplificarlo y a enfatizar supuestos déficits en la oferta laboral, en lugar de reconocer 
el cambio en los patrones de demanda; también existe una confusión acerca de la relación entre hikikomori y las nuevas categorías utilizadas para describir la falta de compromiso de los jóvenes y las nuevas inseguridades del mercado laboral (2008, p. 318). En este sentido, Tanaka afirma:

El fenómeno, tratado al principio como un capricho de jóvenes sostenidos por los padres, quienes les permitían vivir una vida a su antojo, pronto se convirtió en un problema social, económico y político. Fue una respuesta de los jóvenes a la sociedad que los valoraba sólo como mercancía en el mercado de trabajo; fue una variante de hikikomori ante el trabajo (2016, p. 349).

Por su parte, Teo y Gaw hacen una dura analogía entre la leyenda sintoísta en la que la diosa Amaterasu se encerró en una cueva por la vergüenza causada por uno de sus hermanos, haciendo que la oscuridad consumiera a Japón; y dice: "con la sociedad japonesa de hoy está pasando lo mismo, tal vez miles de jóvenes se están encerrando en sus propias cuevas virtuales" (2010, p. 178). En su análisis, Teo y Gaw afirman que los estudios hasta hoy son limitados, particularmente porque el número de afectados no ha sido debidamente calculado y las cifras provienen más de estimaciones, lo que minimiza el fenómeno fundamentalmente por dos razones: a) en las estimaciones se incluyen sólo a los hikikomori que atendieron la llamada de los centros de salud y bienestar del gobierno, pero no se consideran los casos registrados en clínicas y hospitales, $\mathrm{y} b$ ) por cada caso conocido es posible que exista otro, considerando que un hikikomori por definición pretende permanecer aislado (2010, p. 180 y 181).

Umeda y Kawakami (2012), con datos de la encuesta The World Mental Health. Japan Survey Group 2002-2006, hicieron un análisis en el que asociaron la experiencia de vida de un hikikomori con el ambiente en el que se desarrolló durante su infancia, identificando la preexistencia de desórdenes mentales. Tomando en cuenta no sólo la evolución del niño, sino las condiciones de vida de los padres, de quienes consideraron: su nivel educativo, así como la existencia de afecciones mentales. Los autores encontraron una correlación entre la tendencia a la reclusión de hijos de madres que padecen accesos de pánico y padres con un nivel educativo alto. Sugieren que la personalidad introvertida cumple un papel determinante, combinándose negativamente personalidad y ambiente.

En el mismo año, Kato et al. (2012) analizan también el ambiente que rodea a los hikikomori y afirman que hay una influencia de la cultura, la so- 
ciedad y la historia en los desórdenes mentales, encontrando que en Japón, la rapidez de los cambios ha impactado en los jóvenes.

El tránsito de la derrota de la Segunda Guerra Mundial a ser la segunda economía en el mundo; y de ser modelo de desarrollo y avance tecnológico, al estallido de la economía burbuja, han propiciado ambientes de incertidumbre en los jóvenes. La aplicación de medidas neoliberales para recuperar la competitividad japonesa cancela oportunidades que antes brindó.

Es particularmente relevante el tema laboral; la época de empleo ideal terminó. Ante la pérdida de competitividad, las empresas promovieron una reestructuración disminuyendo el número de empleados en sus áreas internas y trasladando a algunos a sus filiales en el exterior; también disminuyeron las horas laborales, contratando a empleados temporales. Las inversiones empresariales en la capacitación de su personal que incentivaban lealtad, estabilidad y rendimiento ya no eran posibles ante la necesidad de reducir costos y mantenerse competitivos. Los cambios en la ley laboral de 2003 (Ley de Estándares Laborales) y el nuevo contrato laboral de 2008 (Ley de Contrato Laboral), así como la exigencia de patrones de formación flexibles y capaces de adaptarse al ambiente, se convirtieron en un reto para la juventud japonesa formada en programas rígidos.

De acuerdo con Suwa y Suzuki, el porcentaje de trabajadores dedicados a la industria manufacturera ha disminuido; en 1975 la tasa de trabajadores en este sector era de $25 \%$, para el año 2010 fue de $16.8 \%$, creciendo rápidamente el sector terciario, requiriendo trabajadores con una preparación diferente, demandando mayores niveles de habilidad y enfrentando una fuerte competencia (2013, p. 191).

Asimismo, los empleos irregulares sin garantía de permanencia ni posibilidad de escalar posiciones se han incrementado. La precarización laboral en Japón es un problema social que afecta a jóvenes, impulsando conductas de aislamiento ante la desesperanza de no poder realizarse profesionalmente.

En el análisis de Kondo et al. (2008) se incluyeron 337 individuos afectados por esta conducta. A diferencia de los autores anteriormente revisados, parten de la necesidad de incluir el diagnóstico y análisis de los problemas mentales individuales. De manera que en los casos estudiados, los sujetos se diagnosticaron con esquizofrenia, con trastornos anímicos o de ansiedad, requiriendo asistencia médica, y en otros sólo apoyo psicosocial por presentar cuadros de trastornos de la personalidad. Asimismo, concluyeron que los casos hikikomori responden a afecciones mentales y pueden diagnosticarse utilizan- 
do los criterios existentes dentro de la especialidad. Lo importante, afirman, es buscar ayuda para evitar empeorar y llegar a un aislamiento prolongado.

Los estudios revisados sobre el tema comparten la premisa de que la atención temprana es fundamental, que debe haber una evaluación psicológica, pero no se deben dejar de lado el contexto social y los antecedentes familiares. La constante entre los autores revisados dice que los factores sociales del Japón de hoy influyen en esta conducta de manera importante. La familia, la escuela, el trabajo, todos los ambientes están imponiendo nuevos ritmos de asimilación. Horiguchi afirma que el mercado laboral, la familia (incluyendo su tamaño), las escuelas y los servicios de atención psicológica han cambiado, haciendo que el síndrome hikikomori, así como se conceptualiza actualmente, haya pasado inadvertido. Por lo tanto, es necesario distinguir entre el comportamiento individual del sujeto aislado y el significado socialmente construido, atendiendo a la posible problematización de ese aislamiento (2012, p. 124). Conocer el contexto ayudará a la definición del nivel de afección, sus probables causas y a diseñar programas de apoyo. De manera que Horiguchi propone utilizar la perspectiva conductual y la psicológica para analizar el fenómeno hikikomori. En la primera, considera conductas como el rechazo a la escuela, la violencia intrafamiliar, la pertenencia a grupos de subculturas (otakus); en la segunda ubica el rechazo a la socialización vinculado a problemas de índole mental (2012, p. 124).

Suwa y Suzuki (2013) afirman que la tendencia al aislamiento es un síndrome que atiende a una patología particular, por lo tanto, deberá ser analizado en función de sus propias características. Basados en el análisis de lo que denominan un hikikomori primario, se podrían entender los problemas sociológicos del Japón de hoy. El fenómeno hikikomori no es sólo un problema de conducta en adultos jóvenes, es también un problema social del Japón contemporáneo (Suwa \& Suzuki, 2013, p. 194).

Para los autores ni las brechas de tiempo, ni el sistema educativo, ni la revolución de las tecnologías de información son causas directas del fenómeno hikikomori; deben considerarse, dicen, tres aspectos importantes en Japón: 1) cambios en la familia; 2) cambios en los grupos sociales, y 3) cambios en el mercado laboral. Dichos cambios no han sido acompañados con una estructura que permita asimilar el cambio y adecuarse a él. El ideal del esfuerzo recompensado con reconocimiento y éxito pierde sentido en una sociedad que trata de responder a las demandas externas con un modelo de organización social que no ha creado las condiciones para asumir que Japón cambió. 
Suwa y Suzuki (2013) plantean que Japón ha vivido etapas de intenso trabajo; una, posterior a la Segunda Guerra Mundial, en la que buscaron la riqueza material y anhelaron los ideales de libertad y éxito económico de Estados Unidos; otra, en la que confiaron en un futuro esperanzador experimentando un gran cambio, reconocida como una etapa de auge con una economía boyante y alto reconocimiento internacional. No obstante, el rápido desarrollo industrial impactó en las relaciones familiares, la crisis cambió todo drásticamente. Poco a poco Japón entró en una etapa de ficción. Los jóvenes a finales de la década de 1970 y principios de 1980 fueron llamados "Shin-jinrui" (nuevos seres humanos) cercanos a la realidad de la prosperidad japonesa; las crisis actuales llevaron a los jóvenes a acercarse más a un mundo virtual que les permitía alejarse de la realidad (Suwa \& Suzuki, 2013, p. 196).

Uchida y Norasakkunkit (2014), al igual que Kondo et al. (2008), afirman que el problema más serio de un hikikomori es no reconocer que sufre de esta afección y buscar ayuda inmediata. El panorama es más complejo cuando, quienes sí buscan ayuda no la reciben de forma adecuada; a menudo por desconocimiento de los patrones particulares de este fenómeno, los afectados son mal diagnosticados y catalogados como agorafóbicos, a pesar de que la motivación de aislamiento de un hikikomori es la de evitar posibles interacciones sociales con individuos que podrían juzgarlos.

Categorizados en ocasiones como autistas o enfermos mentales, se deja de lado un serio problema asociado al desfase entre las prácticas institucionales japonesas dominantes y las presiones postindustriales que pretenden hacer más competitivo a Japón en el mercado global (Uchida \& Norasakkunkit, 2014, pp. 930-931). Japón se enfrenta a una práctica institucional contradictoria que genera un alto costo social, flexibiliza sus normas laborales para enfrentar la competitividad internacional, desaparece garantías como el reconocimiento a la antigüedad, pero no ofrece programas que apoyen a los jóvenes para paliar con los cambios.

Los jóvenes japoneses se sienten impedidos sistemáticamente de lograr una vida social segura y estable, y en ocasiones rechazan los valores culturales con los que fueron formados. Este patrón es consistente con los hallazgos previos en estudiantes de alto riesgo que se desviaron de las tendencias japonesas convencionales para enfocarse más en una visión individual, renunciando a relaciones con estudiantes que siguen los convencionalismos de la sociedad japonesa (Uchida \& Norasakkunkit, 2014, p. 930 y 931). De manera que el fenómeno hikikomori podría ser considerado producto de la percepción de 
los jóvenes en torno a la marginación provocada por la falta de condiciones para realizarse.

Aunque los autores coinciden en que el término hikikomori se refiere a un fenómeno de aislamiento social igual o mayor a seis meses, el término es mejor entendido en relación con sus síntomas psicológicos. Es dentro de esta disciplina en la que se han hecho mayores estudios. Las publicaciones aquí revisadas se ubican en este enfoque; sin embargo, todos asumen que las condiciones sociales que vive Japón hoy influyen determinantemente en la actitud de los jóvenes. Se puede observar que los diagnósticos son diferenciados. Los autores incluyen desde afecciones de personalidad, depresión, hasta enfermedades mentales como la esquizofrenia, pero hay coincidencia en dos temas: a) se requiere un análisis más profundo del fenómeno que defina características propias del hikikomori para proporcionar la ayuda adecuada, y b) los cambios sociales no han sido acompañados de los instrumentos necesarios para que los jóvenes se adapten a su entorno.

\section{Hikikomori. Un fenómeno japonés}

¿Por qué en Japón? Dos espacios han sido relevantes para detonar la conducta de aislamiento social en Japón. En primer lugar la escuela. Niños y jóvenes que se sienten agredidos, presionados y que no encajan en el ambiente escolar prefieren permanecer en casa y gradualmente se alejan de la convivencia social, limitan la comunicación con sus amigos, compañeros e incluso con la familia. En segundo lugar, está el espacio laboral. La falta de oportunidades acordes con su formación y con recompensas a su esfuerzo los lleva recluirse en sus habitaciones.

La escuela es uno de los factores que influyen en el aislamiento social. De acuerdo con Sugimoto, el estricto régimen educativo japonés caracterizado por prácticas que siguen una ética militar, la uniformidad psicológica para favorecer la cohesión entre los estudiantes, un estricto control por parte del profesorado y acciones colectivas en donde un grupo fuerte humilla a otro que se encuentra en situación de desventaja (ijime o bullying) provoca respuestas tales como fobia escolar, absentismo y reclusión (Sugimoto, 2014, pp. 146-149).

Aunque los temores y conflictos emocionales suelen ser una etapa común en los adolescentes por lo que representa el tránsito hacia las responsabilidades del adulto, en Japón, como afirman Uchida y Norasakkunkit (2014), los jóvenes además enfrentan un desfase entre las prácticas institucionales 
y las presiones postindustriales para hacer un Japón más competitivo. Los jóvenes japoneses se sienten paralizados por profundos temores sociales, el compromiso con el éxito, el deseo de iniciar una vida con sus propios recursos se convierte en una meta que se frustra al no contar con las herramientas para superar el reto. Las instituciones educativas proporcionan excelentes programas de formación, pero éstos ya no responden a las necesidades del campo laboral ni tampoco ofrecen capacitación para paliar con el reto que representa transitar de la etapa de formación a la laboral. "Tengo toda clase de emociones negativas dentro de mí, ira hacia la sociedad, a mis padres; tristeza por vivir en estas condiciones, temor al futuro y celos de las personas que llevan una vida normal" (Declaración de un hikikomori en Kremer y Hammond, 2013).

Jóvenes perdidos en la transición de la escuela al empleo, de la adolescencia a la adultez vinculada a una profunda transición de la sociedad japonesa, son etapas de vida asociadas a lo que Mary C. Brinton denomina "locaciones sociales", contextos en los que los individuos encuentran seguridad e identidad; y en donde el cambio en las instituciones sociales deberá ser acompañado de herramientas para enfrentarlos (2011, p. 2). Brinton afirma que las escuelas japonesas formaban a egresados exitosos, les proporcionaban herramientas útiles para ubicarlos laboralmente en corporaciones prestigiosas, asegurándoles reputación y tranquilidad en su vida (2011, p. 3).

Fuerzas sociales como la reputación que una persona adquiere en la sociedad (seketei), implican una presión constante sobre el individuo para cumplir con los compromisos que la hagan formar parte de un grupo a partir del reconocimiento individual y no convertirse en un fracaso social (Kremer \& Hammond, 2013). Amae o dependencia indulgente es en Japón la necesidad de depender y confiar sumisamente de una persona, todas las relaciones deben fincarse en esta confianza para fortalecer el grupo. Los padres promueven esta dependencia, a cambio de años de apoyo, los padres esperan respeto y honor que provenga del reconocimiento que la sociedad otorga a su hijo al conseguir un buen trabajo (Doi, 2001, p. 11-26).

Davies e Ikeno dirían que amae es el medio vital para relacionarse con otros, es la base para mantener relaciones armónicas; los hijos dependen de los padres, los jóvenes de los adultos, los abuelos de los nietos mayores y así; de manera que el concepto amae afecta todos los aspectos de las relaciones sociales en Japón (Davies e Ikeno, 2002, pp. 17-18). El no sentirse parte de este medio vital empuja a los jóvenes a buscar alternativas en donde no les juzguen ni demanden ese reconocimiento que esperan de ellos. 
A mediados de la década de 1990, en esta sociedad que Ohsawa (2008) etiquetó como "la agencia de la tercera persona" o persona trascendental que puede juzgar la realidad y apropiarse de estándares sociales se vuelve hoy imprescindible; sin esta tercera persona nadie garantiza la validez de las normas sociales o estándares y los individuos, sin una guía hacia el éxito, se ven obligados a decidir solos sobre su propio futuro. Esta tercera persona, traducida en instituciones y líderes, fue un factor determinante para construir una sociedad fuerte y cohesionada, desenvolviéndose en el marco de planes económicos que generaron certidumbre.

Finalmente, en un país con una sociedad basada en la veteranía, las principales instituciones sociales sugieren que, a medida que Japón envejezca aún más, las demandas y necesidades de sus jóvenes serán menos atendidas, ya que la demanda de atención de un envejecido sector de la población requerirá mayor tiempo y recursos.

\section{Las acciones del gobierno japonés}

Los primeros reportes del Ministerio de Salud, Trabajo y Bienestar de Japón (MHLW, por sus siglas en inglés) se publicaron en los años 2001, 2003, 2010 y 2016. En el año 2003 el MHLW publicó un libro blanco sobre el tema y definió al fenómeno hikikomori como un estado en el que persiste el aislamiento social por seis meses o más y que puede deberse a la dificultad de adaptación a su entorno; aunque no se descartan los factores biológicos, de manera que se entrelazan factores psicológicos y sociales (Ministry of Health, Labour and Welfare, 2003).

En el año 2010 la llamada "Encuesta sobre la conciencia de los jóvenes (condiciones reales de un hikikomori)" publicada por la Oficina del Gabinete del gobierno de Japón (Japan Cabinet Office, 2010), incluyó 37 preguntas para evaluar el fenómeno, entre ellas destacan las que evalúan el entorno: número de personas con las que vive, experiencia laboral, tiempo y actitud frente al trabajo, frecuencia con la que sale, deseo de conversar con alguien sobre sus problemas. Las encuestas se distribuyeron aleatoriamente. Se recuperaron completas 3,287, de ellas el grupo identificado como hikikomori se compuso de 59 individuos (1.79\%).

En el mes de septiembre de 2016 se publicaron los resultados de la encuesta "La Vida de los Jóvenes", que incluyó jóvenes y adultos entre 15 y 39 años. En ella el gobierno japonés reconoce la necesidad de crear mecanismos 
para apoyar a los jóvenes afectados y realizar estudios más completos a fin de identificar los factores que impactan directamente en su comportamiento. La encuesta es importante porque reconoce que ser un hikikomori no es sólo un tema de salud mental, sino que asume que el ambiente social es un factor que también puede influir en estas conductas (Japan Cabinet Office, 2016).

El número de respuestas validadas de la encuesta de 2016 fue de 2,905, de las cuales sólo el $1.69 \%$ reconocieron la condición de hikikomori, de ellos más del 60\% son hombres y casi el 40\% mujeres (Japan Cabinet Office, 2016; Tajan, Yukiko, \& Pionnié-Dax, 2017).

Aunque el número de personas recluidas disminuyó de 696 mil en 2010 a 541 mil en 2016, el problema persiste, de manera que el MHLW se ha dado a la tarea de realizar otros estudios para emprender acciones concretas. De las acciones encaminadas a reforzar la atención a los hikikomori se encuentran las encuestas realizadas en los Centros de Apoyo Regional a hikikomori. De cinco centros regionales encuestados se derivaron cuatro iniciativas: 1) sumar esfuerzos para encontrar opciones viables; 2) realizar más consultas; 3) definir apoyo a miembros de la familia de un hikikomori, y 4) brindar apoyo gradual al afectado. Lo más importante de estos estudios es la visibilización del fenómeno y el compromiso de ampliar las redes de apoyo y la asesoría a organizaciones vinculadas con la atención a este sector (MHLW, 2016).

En el presupuesto fiscal de 2018, el gobierno de Japón ha destinado 20 millones de yenes para financiar la primera encuesta nacional para hikikomori de mediana edad. La encuesta inició en abril de 2018 y se realiza en respuesta a la solicitud de ayuda de padres que atienden hijos sin trabajo, dependientes desde el punto de vista financiero y socialmente recluidos; ésta cubrirá alrededor de 5,000 hogares con miembros en edades entre los 40 y 59 años de edad (Japan Times of News, 2018).

Con esta encuesta se pretende encontrar las causas de la reclusión social en este sector de la población, especialmente importante por encontrarse en edad laboral, en la etapa cuando la persona alcanza la satisfacción de vida. Se busca saber si existen causas familiares que influyan en las personas para no tener contacto con la sociedad y renunciar al trabajo, o bien saber si es el ambiente externo, como el centro laboral el que no cubría las expectativas de la persona. Los resultados pueden arrojar datos sobre otros factores que afecten a este sector poblacional diferentes a aquéllos detectados en las encuestas anteriores. El gobierno tomó esta iniciativa considerando que cada vez más los recluidos están envejeciendo en su encierro. "Mientras que los 
casos de personas recluidas en sus hogares por menos de seis meses fueron de 696,000 en 2010 a 541,000 en 2015, la proporción de aquellos que se han aislado de la sociedad por siete años o más aumentó de 16.9 a 34.7\%, según los datos del gobierno" (Japan Times of News, 2018).

Asimismo, los resultados de esta encuesta permitirán saber la edad en que las personas decidieron recluirse, dando luz sobre los factores contextuales que abonaron para tomar esa decisión, además de saber si han sido apoyados por la familia, o bien si no han encontrado estímulos para dejar la reclusión.

\section{Comentarios finales}

Japón atraviesa por un periodo de cambio; transita de una sociedad de ideales y sueños caracterizados por un ambiente de realización plena, a una etapa de recomposición social que afecta particularmente a jóvenes con aspiraciones, pero desprovistos de los medios para realizarlas.

De acuerdo con los estudios aquí revisados el síndrome de hikikomori ha estado presente en la sociedad japonesa desde hace tiempo; autores reportan síntomas desde la década de 1950. Los análisis se elaboran desde la psicología y es en el marco de esta disciplina que el fenómeno ha cobrado relevancia y se ha tratado de explicar. Sin embargo, todos los autores revisados consideran dos factores concomitantes: el contextual, en donde el ambiente que les rodea no les brinda los elementos para satisfacer sus aspiraciones, y el estructural, en el que se encuentran instituciones como la familia y la escuela, cuyos códigos siguen siendo rígidos de frente a una sociedad que demanda flexibilidad.

Dos elementos destacan: la falta de comunicación familiar y el reconocimiento de los síntomas. Generalmente, coinciden los autores, inicia con una fobia a la escuela o al espacio laboral, apatía, ensimismamiento, frustración, y en ocasiones estas conductas se desestiman porque se atribuyen a un estadio de la adolescencia o de la rebeldía del tránsito hacia la adultez; sin embargo, se ha dejado de lado el problema que representa la interconexión entre individuos y familia y entre la sociedad y la estructura institucional.

El fenómeno ha sido más visibilizado a partir de que el gobierno asumió el fenómeno hikikomori como un problema de la sociedad japonesa y decidió realizar estudios que le permitieran llevar a cabo acciones concretas. No obstante, avanzar en su atención ha sido complicado debido a dos situaciones: en su mayoría quienes han decidido recluirse no desean dar a conocer su situación, por lo tanto es difícil tener un número confiable de quienes pueden ser identi- 
ficados como hikikomori, y para el gobierno es un asunto delicado, toda vez que implica asumir la responsabilidad frente a los cambios que ha emprendido sin considerar las condiciones sociales y culturales de su propia sociedad.

Es importante dar a conocer este tipo de fenómenos que se extienden en la medida que se cancelan oportunidades para la realización de los jóvenes; lo vertiginoso de los cambios sociales y económicos en el mundo está dejando desprovistos a grandes sectores de la población de las herramientas para asumirse como miembros de la sociedad y encontrar incentivos para realizarse. niv

\section{Referencias bibliográficas}

Brinton, M. C. (2011). Lost in Transition. Youth, Work, and Instability in Postindustrial Japan. New York: Cambridge University Press.

Davies, R.J. \& Ikeno, O. (2002). The Japanese Mind. Understanding Contemporary Japanese Culture. Tokyo: Tuttle Publishing.

Doi, T. (2001). The anatomy of dependence: The key analysis of Japanese behavior. Tokyo: Kodansha International Ltd.

Erikson, E. H. (1950). Childhood and Society. New York-London: Norton and Company.

Furlong, A. (2008). The Japanese Hikikomori Phenomenon: Acute Social Withdrawal among Young People. The Sociological Review, 56(2), 309-325. doi: 10.1111/j.1467-954X.2008.00790.x

Horiguchi, S. (2012). Hikikomori: How Private Isolation Caught the Public Eye. In R. Goodman, Y. Imoto, \& T. Toivone (Eds.), A Sociology of Japanese Youth: From Returnees to NEETs (pp. 122-138). London \& New York: Routledge.

Japan Cabinet Office. (2010). wakamono no ishiki ni kansuru chousa (hikikomori ni kansuru jittai chousa) [Survey of actual conditions of hikikomori] Abridged version. Recuperado de https://www8.cao. go.jp/youth/kenkyu/hikikomori/pdf_gaiyo_index.html

Japan Cabinet Office. (2016). heisei 25 nendo waga kuni to shogaikoku no wakamono no ishiki ni kansuru chousa [La Vida de los Jóvenes" que incluyó jóvenes y adultos entre 15 y 39 años] Recuperado de https://www8. cao.go.jp/youth/kenkyu/thinking/h25/pdf_index.html

Japan Times of News. (7 de enero de 2018). Japan's first nationwide survey of middle-aged hikikomori in the pipeline. Japan Times of News. Recuperado 
de https://japan.timesofnews.com/japans-first-nationwide-survey-ofmiddle-aged-hikikomori-in-the-pipeline.html

Kato, T.A., Tateno M., Shinfuku N., Fujisawa D., Teo AR., Sartorius N., ..., Kanba S. (2012). Does the 'hikikomori' syndrome of social withdrawal exist outside Japan? A preliminary international investigation. Social Psychiatry and Psychiatric Epidemiology, 47(7), 1061-1075. Recuperado de https:/www.ncbi.nlm.nih.gov/pmc/articles/PMC4909153/ doi:10.1007/ s00127-011-0411-7

Kondo N., Miyazawa H., Sakai M., Kiyota Y., Kitahata Y., ... Miyata R. (2008). Shishunki hikikomori ni okeru seishin igakuteki shougai no jittai haaku ni kan suru kenkyuu [Research on the psychiatric disorders in adolescents with social withdrawal] Kokoro no kenkou kagaku kenkyuu. Citado en Teo A. \& Gaw, A. (2010). Hikikomori, a Japanese Culture-Bound Syndrome of Social Withdrawal? A Proposal for DSM-5. The Journal of Nervous and Mental Disease, 198(6), 444-449. doi: 10.1097/NMD.0b013e3181e086b1

Kremer, W., \& Hammond, C. (5 de julio de 2013). Hikikomori: Why are so many Japanese men refusing to leave their rooms? BBC News. Recuperado de http://www.bbc.com/news/magazine-23182523

Ministry of Health, Labor and Welfare (MHLW). (2003). The concept of hikikomori. Recuperado de https://www.mhlw.go.jp/topics/2003/07/ tp0728-1b.html

Ministry of Health, Labor and Welfare (MHLW). (2016). A Survey on the Project for the Establishment and Operation of Regional Support Centers for Hikikomori, (Abnormal Avoidance of Social Contact) Labor and Welfare. Recuperado de http://www.mhlw.go.jp/stf/houdou/0000116367.html

Ohsawa M. (2008). Fukanousei no Jidai. Tokyo: Iwanami Shin-syo (in Japanese). Citado en Suwa, M. \& Suzuki, K. (2013). The phenomenon of "hikikomori" (social withdrawal) and the socio-cultural situation in Japan today. Journal of Psychopathology 19, 191-198. Recuperado de http://www. jpsychopathol.it/issues/2013/vol19-3/01b-Suwa.pdf

Saito, T. (2013). Hikikomori. Adolescence without End. [Hikikomori Adolescencia sin fin]. Estados Unidos: University Of Minnesota Press

Sugimoto, Y. (2014). An Introduction to Japanese Society. (4 ed.). Reino Unido: Cambridge University Press. Recuperado de http://assets.cambridge. org/97811076/26676/frontmatter/9781107626676_frontmatter.pdf

Suwa, M., \& Suzuki, K. (2013). The phenomenon of "hikikomori " (social withdrawal) and the socio-cultural situation in Japan today. Journal of 
Psychopathology 19, 191-198. Recuperado de http://www.jpsychopathol. it/issues/2013/vol19-3/01b-Suwa.pdf

Tajan, N., Yukiko H., \& Pionnié-Dax, N. (marzo, 2017). Hikikomori: The Japanese Cabinet Office's 2016 Survey of Acute Social Withdrawal. The Asia Pacific Journal, 15(5). Recuperado de http://apjjf.org/2017/05/Tajan.html Tanaka, M. (2016). La juventud japonesa ante la crisis del neoliberalismo. Estudios de Asia y África 51(2), 329-363. Recuperado de https://estudiosdeasiayafrica.colmex.mx/index.php/eaa/article/view/2173 doi: 10.24201/eaa.v51i2.2173

Teo, A. R., \& Gaw, A. C. (junio, 2010). Hikikomori, a Japanese CultureBound Syndrome of Social Withdrawal? A Proposal for DSM-5. The Journal nervous and mental disease, 198(6) 444-449. doi: 10.1097/ NMD.0b013e3181e086b1

Uchida, Y., \& Norasakkunkit V. (2014). To Conform or to Maintain SelfConsistency? Hikikomori Risk in Japan and the Deviation From Seeking Harmony. Journal of Social and Clinical Psychology, 33(10), 919-937. doi: 10.1521/jscp.2014.33.10.918_

Umeda, M., \& Kawakami, N. (marzo, 2012). Association of childhood family environments with the risk of social withdrawal ('hikikomori') in the community population in Japan. Psychiatry and Clinical Neurosciences, 66(2), 121-129. doi: 10.1111/j.1440-1819.2011.02292.x 